\title{
Ilixadencel - an Allogeneic Cell-Based Anticancer Immune Primer for Intratumoral Administration
}

\author{
Alex Karlsson-Parra ',2 - Juliana Kovacka' - Emilia Heimann ' Margareth Jorvid '. \\ Sijme Zeilemaker ${ }^{\prime} \cdot$ Sharon Longhurst ${ }^{\prime} \cdot$ Peter Suenaert ${ }^{\prime}$
}

Received: 27 April 2018 / Accepted: 1 June 2018 / Published online: 14 June 2018

(C) The Author(s) 2018

\begin{abstract}
Intratumoral administration of an immune primer is a therapeutic vaccine strategy aimed to trigger dendritic cell (DC)-mediated cross-presentation of cell-associated tumor antigens to cytotoxic $\mathrm{CD}^{+} \mathrm{T}$ cells without the need for tumor antigen characterization. The prevailing view is that these cross-presenting DCs have to be directly activated by pathogen-associated molecular patterns (PAMPS), including Toll-like receptor ligands or live microbial agents like oncolytic viruses. Emerging data are however challenging this view, indicating that the cross-presenting machinery in DCs is suboptimally activated by direct PAMP recognition, and that endogenous inflammatory factors are the main drivers of DC-mediated cross-presentation within the tumor. Here we present preclinical mode of action data, CMC and regulatory data, as well as initial clinical data on ilixadencel. This cellbased drug product is an off-the-shelf immune primer, consisting of pro-inflammatory allogeneic DCs secreting high amounts of pro-inflammatory chemokines and cytokines at the time of intratumoral administration. The mechanism of action of ilixadencel is to induce recruitment and activation of endogenous immune cells, including NK cells that subsequently promotes cross-presentation of cell-associated tumor antigens by co-recruited DCs.
\end{abstract}

KEY WORDS cross-presentation · dendritic cell . intratumoral · ilixadencel · NK cell

Guest Editors: Karin Hoogendoorn and Christopher A. Bravery

Alex Karlsson-Parra

alex.karlsson-parra@immunicum.com

Immunicum AB, Grafiska Vägen 2, 41263 Gothenburg, Sweden

2 Department of Immunology, Genetics and Pathology, Rudbeck Laboratory, Uppsala University, Dag Hammarskjölds Väg 20, 752 37 Uppsala, Sweden

\author{
ABBREVIATIONS \\ ATMP Advanced Therapy Medicinal Product \\ CDCl Conventional type 1 DCs \\ CPI Check Point Inhibitors \\ CTL Gytotoxic T Lymphocytes \\ DC Dendritic Cell \\ HLA Human Leukocyte Antigen \\ IFN Interferon \\ IL Interleukin \\ INN International Nonproprietary Name \\ MDSC Myeloid-Derived Suppressor Cells \\ MoDC Monocyte-derived Dendritic Cell \\ NK Natural Killer \\ PBMC Peripheral Blood Mononuclear Cells \\ PD-I Programmed Cell Death Protein-1 \\ PD-LI Programmed Cell Death-Ligand 1 \\ TAP Transporter associated with Antigen Processing \\ TLR Toll-Like Receptor \\ TME Tumor Microenvironment \\ TNF Tumor Necrosis Factor
}

\section{INTRODUCTION}

Agents known as immune check point inhibitors (CPIs), particularly therapeutic antibodies that block the immunosuppressive programmed cell death protein-1 (PD-1) and the programmed cell death-ligand 1 (PD-L1) pathway, are now revolutionizing the practice of medical oncology due to their ability to improve outcomes in various malignancies. In contrast, with the exception of some forms of premalignant disease, the proportion of patients benefiting from treatment with cancer vaccines leaves much to be desired [1].

In most mouse models, anti-tumor immunity requires the generation of tumor-specific cytotoxic $\mathrm{CD}^{+} \mathrm{T}$ cells (CTLs) and it is similarly believed that CTLs are major players in successful immunotherapies of human cancers. There is now 
emerging evidence that PD-1/PD-L1 inhibitors are more effective in tumors that are already recognized by the immune system, as manifested by a pre-existing $\mathrm{CD}^{+}{ }^{+}$-cell infiltrate [2], indicating that anti-PD-1/PD-L1 antibodies reactivate disabled intratumoral $\mathrm{T}$ cells [3]. In patients with no preexisting antitumor $\mathrm{T}$ cells, a vaccine-induced reactivation of tumor-specific $\mathrm{T}$ cells and also activation of $\mathrm{T}$ cells from the naive repertoire is therefore a rational option to counteract clinical resistance to CPIs [4]. Indeed, recent findings indicate that the lack of spontaneous immune infiltration in solid tumors is unlikely due to a lack of tumor-specific antigens that potentially may be recognized by the immune system [5]. The vaccine approach could thus potentially transform a noninflamed" cold" non-permissive tumor resistant to CPIs into a sensitive inflamed" hot" tumor [6]. Combining cancer vaccines with CPIs or other anti-cancer drugs, like gemcitabine and sunitinib, known to counteract myeloid-derived suppressor cell (MDSC)-derived immunosuppression [7] may thus provide the necessary stimulation to broaden the repertoire of $\mathrm{T}$ cells engaged in the antitumor response.

\section{Mutation-Derived Tumor Antigens (Neoantigens)}

Effective anti-tumor immunity induced by CPIs or adoptive $\mathrm{T}$ cell therapy in humans has been associated with the presence of $\mathrm{T}$ cells directed at cancer neoantigens [8, 9], a class of human leukocyte antigen (HLA)-bound peptides that arise from tumor-specific mutations $[10,11]$. These mutationderived and patient-specific neoantigens are usually highly immunogenic because they are not present in normal tissues and hence bypass central thymic tolerance [12]. These facts have breathed new life into the field of cancer vaccines, and treatment with neoantigen-based vaccines, in which the patient's neoantigens are first characterized and then synthesized in vitro, is presently undergoing several clinical trials and initial clinical data are indeed promising $[13,14]$.

However, on a purely practical level, the manufacturing process of neoantigen-based vaccines includes many obstacles that will need to be overcome. In addition, this production is entirely patient dependent, i.e. can only be performed after the neoantigens for each individual patient have been characterized from a tissue sample taken from the patient's own tumor which constitutes quite a logistical challenge [10, 11].

\section{Intratumoral (In Situ) Administration of Immune Primers}

A rational way to circumvent practical problems associated with characterization and in vitro production of tumor neoantigens is to use the patient's existing tumor (or metastasis of) as a direct neoantigen source by injecting an immune primer directly into the patient's own tumor. Such an approach would allow for the development of vaccines in patients themselves, thereby minimizing the resource allocation required in ex vivo processing. Furthermore, this strategy may take advantage of the complete neoantigen repertoire of the patients tumor rather than be limited to a restricted number of characterized and ex-vivo produced tumor neoantigens [15].

\section{The Immunosuppressive Tumor Microenvironment}

The tumor microenvironment (TME) contains stromal cells and immune cells that shape cancer development and impact the response to tumor therapy [16]. Intratumoral immune cells comprise lymphocytes, such as T cells, and natural killer (NK) cells, and diverse populations of myeloid cells, including MDSC, macrophages, and dendritic cells (DCs) [16]. Simplistically, intratumoral MDSCs, M2-polarized macrophages and regulatory $\mathrm{CD} 4{ }^{+} \mathrm{T}$ cells (Treg) can promote cancer cell growth, angiogenesis, and metastasis, as well as contribute to the establishment of an immunosuppressive environment. The presence of these cells within the tumor is associated with tumor progression and poor clinical outcome [17]. Additionally, tumor stromal fibroblasts have recently been shown to be major producers of immunosuppressive TGF- $\beta$ that inhibits $\mathrm{T}$ cell recruitment into the tumor [18, 19], thus potentially explaining why certain tumors with a high mutational load still lack infiltrating T cells [20].

\section{Conventional Type I DCs}

It is well understood that antigen-presenting cells within tumors typically do not maintain cytotoxic $\mathrm{CD}^{+} \mathrm{T}$ cell (CTL) function, despite engaging them. Across multiple mouse tumor models and human tumor biopsies, intratumoral conventional type 1 DCs (cDC1), bearing CD103 in mouse and CD141 in humans, are extremely sparse and yet remarkably capable stimulators of CTLs [21, 22]. These are uniquely dependent upon Batf3 transcription factors and generated by GM-CSF and Flt3L cytokines. Regressing tumors have higher proportions of these cells, T-cell dependent immune clearance relies upon them, and abundance of their transcripts in human tumors correlates with clinical outcome $[21,22]$. The cDC1 subset is especially adapted at taking up cell-associated antigens from dying tumor cells and transporting tumor-derived antigens to tumor-draining lymph nodes where they constitute the key DG subtype responsible for cross-presentation of tumor-derived antigens to tumorspecific $\mathrm{CD}^{+} \mathrm{T}$ cells $[22,23]$. In addition to this trafficking role, cDCl also play a key role within tumors themselves by re-stimulating and expanding tumor-specific $\mathrm{CD}^{+} \mathrm{T}$ cells [21], and support T cell effector function by secreting interleukin (IL)-12p70 [24]. The overall importance of cDC1 in anti-tumor immunity is underscored by multiple studies demonstrating that the lack of cDC1 in Batf3 knock out mice abolishes the rejection of immunogenic tumors and the 
response to adoptive $\mathrm{T}$ cell therapy and to immune checkpoint blockade [21, 22].

\section{Recruitment of DCs}

Since $\mathrm{cDC} 1 \mathrm{~s}$ are usually very sparse within the tumor, therapies aimed at increasing intratumoral cDC1 abundance are expected to boost anti-tumor immunity and potentially increase the responsiveness of cancer patients to immunotherapy inhibiting tumor-derived immunosuppression [21, 22]. Recently, a key role for intratumoral NK cells was uncovered by their production of chemoattractants, including the chemokine RANTES (also known as CCL5), that are necessary for the accumulation of $\mathrm{cDCl} 1$ in incipient tumors and for tumor immune control in mouse models [25]. Evidence were further provided that a similar NK cell/ chemokine functional axis determines cDC1 abundance in human melanoma, breast cancer, lung cancer, and head and neck squamous cell carcinoma and show that it impacts on patient survival [25].

\section{Induction of Th I-Polarizing Mature DCs}

Different types of immune primer, including different Tolllike receptor (TLR) ligands and pro-inflammatory cytokines, including TNF- $\alpha$ and IL- $1 \beta$, are well-known DC activators. One issue that remains to be fully addressed is the choice of $\operatorname{primer}(\mathrm{s})$ that would appropriately stimulate both DCmediated T-helper 1 (Thl) polarization of tumor-specific $\mathrm{CD}^{+} \mathrm{T}$ cell and cytotoxic $\mathrm{CD}^{+} \mathrm{T}$ cell $(\mathrm{CTL}$ ) responses. Activated/mature DCs are characterized by their expression of membrane-bound co-stimulatory molecules like CD80 and CD86 and may potentially secrete the Th1- and CTLpolarizing factor IL-12p70. The ability to secrete IL-12p70 is, however, not an intrinsic attribute of activated DCs and uncommitted immature DC thus require concomitant exposure to IFN- $\boldsymbol{\gamma}$ when activated by TLR ligands and/or proinflammatory cytokines, to develop the capacity to produce high levels of IL-12p70 upon subsequent contact with helper $\mathrm{T}$ cells expressing the CD40 ligand. This effect is specific for IFN- $\gamma$ and is not shared by other IL-12-inducing factors [26].

\section{Cross-Presentation}

The ability to cross present soluble or cell-associated tumor antigens to tumor-specific $\mathrm{CD}^{+}{ }^{\mathrm{T}}$ cells is key to the antigenpresenting function of mature tumor antigen-loaded DCs. As mentioned earlier, conditions of DC maturation have been shown to be important for DCs ability to activate $\mathrm{T}$ cells by the expression of co-stimulatory molecules and production of the Th1-deviating cytokine IL-12p70. However, it remains partially unknown how different pathways of DC maturation are associated with modulation of their ability to cross present captured exogenous cell-associated tumor antigens. When a minimal peptide epitope is used for antigen-loading of DCs, no additional processing of the peptide is required to generate HLA class I antigen-peptide complexes. In contrast, the generation of such complexes from soluble or cell-associated proteins released from dying tumor cells requires phagocytosis/ endocytosis and processing by the antigen-processing machinery. The cross-presenting pathway requires antigen export of polypeptides from endosomal compartments into the cytosol, proteasomal digestion and transport by the transporter associated with antigen processing (TAP)-subunits TAP1 and TAP2 of polypeptides to the endoplasmic reticulum or endosomes, where final peptide trimming and MHC-I peptide loading take place $[27,28]$. Notably, recent studies indicate that activated NK-cells, by their combined production of IFN- $\gamma$ and TNF- $\alpha$, efficiently induce cross-presentation of cell-associated tumor antigens in human monocyte-derived DCs [29-31] and human CD141 ${ }^{+}$cDC1 [29], the latter observation highlighting a previously unknown cross-talk between NK cells and CD141 ${ }^{+}$DCs for inducing tumor cellderived antigen cross-presentation. In comparative in vitro studies using monocyte-derived DCs (MoDG) [29], it was demonstrated that the concomitant release of IFN- $\gamma$ and TNF- $\alpha$ from activated NK-cells was clearly superior to direct stimulation with TLR3 and TLR7/8 ligands in promoting cross-presentation of cell-associated tumor antigens by monocyte-derived DCs. Stimulation with IFN- $\gamma$ plus TNF- $\alpha$ was also highly efficient in promoting cross-presentation of cell-associated tumor antigens by human cDC1 $\left(\mathrm{CD} 141^{+}\right.$ DCs). The poor or modest induction of cross-presentation in MoDGs by the TLR ligand poly I:C (TLR3 ligand) and R848 (TLR7/8 ligand) is in line with other reports from in vitro studies using MoDCs where single-stimulation with poly I:C (or IFN- $\alpha$ ) did not affect TAP-expression in MoDCs while singlestimulation with IFN- $\gamma$ induced significantly increased expression of both TAP-1 and TAP-2 [32].

At face value, these findings are contradicted by multiple reports from mouse models, indicating that intratumoral injection of TLR ligands like poly I:C is inducing subsequent priming of $\mathrm{CD}^{+} \mathrm{T}$ cells and also challenges the current" dogma" that a DC must become directly activated through pattern recognition receptors, including TLRs, in order to efficiently induce a CTL response. Reis e Sousa and colleagues [33] thus reported that APCs indirectly activated by pro-inflammatory endogenous factors have the capacity to promote the proliferation of naïve $\mathrm{CD}^{+} \mathrm{T}$ cells but that this does not lead to sustained clonal expansion and does not suffice to generate effector T cells endowed with CTL properties. However, since the model tumor antigens that were used in these studies were minimal peptide epitopes, the validity of these observations for cell-associated tumor antigens which have to be cross presented, was not addressed.

Taken together, an intratumorally injected immune primer should ideally be able to induce recruitment of $\mathrm{cDCl}$ and 
induce tumor cell death facilitating the release of cellassociated neoantigens for subsequent capture by recruited DCs [22]. The immune primer should moreover be able to promote DG-mediated cross-presentation of captured cellassociated neoantigens, as well as induce proper DC-activation, in order to induce systemic Thl-deviated anti-tumor $\mathrm{T}$ cell responses, allowing the neoantigen-specific $\mathrm{T}$ cells to attack and destroy the treated tumor and also distant tumor lesions [34]. Finally, an optimal immune primer would preferably also inhibit additional intratumoral immunosuppressive pathways that are not targeted by co-delivered immunosuppression blockers like CPIs.

\section{Intratumorally Injected Allogeneic Pro-Inflammatory DCs as" off-the-shelf" Anticancer Immune Primers}

As to potential potent immune enhancers that can be used for intratumoral administration, recent studies have shown that autologous DCs, which have been pre-activated during a limited time period ex vivo, have the potential to indirectly prime naïve $\mathrm{CD}^{+} \mathrm{T}$ cells in vivo, by acting as a pure immunogenic adjuvant/primer affecting recruited endogenous DCs and not as antigen presenters $[35,36]$. This indirect immune enhancing function of DCs was strictly dependent on their active secretion of certain DC- and NK-cell-recruiting chemokines at the time of administration [35]. In line with these data, preclinical findings indicate that the efficient induction of antigenspecific CTLs, characterizing viral infections, is caused by cross-priming, where initially infected DCs produce a unique set of inflammatory factors that recruit and activate noninfected "bystander" DCs [37-39]. Notably, in the study by Pang et al., signaling through the interleukin 1 receptor (IL$1 \mathrm{R})$ was required for productive cross-presentation of cellassociated viral antigens to $\mathrm{CD}^{+} \mathrm{T}$ cells, while signaling through the pattern recognition receptors TLR7 and RIG-I was not. In line with these findings, a recent study showed that cross-presentation of cell-associated viral antigens by human $\mathrm{CD} 141^{+} \mathrm{cDC} 1$, which are constitutively resistant to viral infection, rely on viral antigens produced in infected bystander DCs [40]. By dissociating viral infection from antigen presentation, this mechanism protects the functional capacity of cDC1 to launch a CTL-mediated adaptive immunity against viral infection.

In vitro studies, using human MoDCs, have further shown that these cells can be programmed to produce vigorous amounts of Th1-associated chemokines and cytokines in a sustained fashion when stimulated with a combination of stimulatory factors, including Toll-like receptor (TLR) ligands and IFN- $\gamma$, thus mimicking virally infected DCs $[41,42]$. Notably, activated MoDCs were found to induce recruitment of NK cells in vitro [42]. When comparing the ability to recruit immunosuppressive $\mathrm{FOXP}^{+}{ }^{+}$regulatory $\mathrm{T}$ cells (Treg) between different DG-types used in the clinical setting, PGE2 matured
DCs, but not type-1 polarized DCs (generated in the presence of the TLR3 ligand poly I:C plus IL- $1 \beta$, TNF- $\alpha$, IFN- $\gamma$ and IFN- $\alpha$ ), showed high Treg-attracting activity [43]. Moreover, in the study by Pang et al., using transgenic mice, it was shown that no MHC compatibility was needed between infected DCs and non-infected, cross-presenting, DCs [39]. By using allogeneic DCs as immune primers in the vaccine setting, such cells will further be regarded as foreign allogeneic invaders that most likely will potentiate a Thl-deviated inflammatory reaction, further promoting recruitment and activation of endogenous" bystander" DCis at the vaccination site [44].

\section{STUDIES WITH PRO-INFLAMMATORY ALLOGENEIC DENDRITIC CELLS}

\section{Preclinical Studies In Vitro}

In a recently published paper [45], the interaction of proinflammatory DCs, activated with a highly potent cocktail consisting of poly I:C (TLR3 ligand), R848 (TLR7/8 ligand) and IFN- $\gamma$ [41] with allogeneic peripheral blood mononuclear cells (PBMCs) (mimicking the interaction of intratumorally injected allogeneic DCs and recruited immune cells from the patient) was investigated. To summarize, pro-inflammatory DCs were found to produce several Th1-associated cytokines and chemokines in a sustained fashion, including IL-12p70 and CXCL-10 (IP-10). The production of pro-inflammatory factors by GMP-produced pro-inflammatory DCs (ilixadencel; see manufacturing process below) stimulated with the same cocktail have further been shown to also produce TNF- $\alpha$, IL- $1 \beta$ and MIP- $1 \alpha$ as well as high amounts of IL12 p70 and RANTES [46]. Moreover, this type of proinflammatory DCs were found to efficiently activate cocultured allogeneic NK cells and T cells and also supported strong cytolytic function of NK-cells. The interaction with allogeneic PBMCs further created a milieu in favor of maturation of immature" bystander" DCs. Finally, when a cellassociated antigen was provided exogenously, supernatants from co-cultures with these pro-inflammatory DCs and allogeneic PBMCs promoted a highly efficient cross-presentation in bystander MoDCs (more than 10-fold increase when compared to non-treated immature MoDCs) of cell-associated tumor antigens to antigen-specific, IFN- $\gamma$ producing $\mathrm{CD}^{+} \mathrm{T}$ cells [45].

\section{Preclinical Studies In Vivo}

In in vivo mouse models, intratumoral administration of allogeneic mouse DG activated with an identical stimulation cocktail as used for human DCs (except that human recombinant IFN- $\gamma$ was replaced by the mouse equivalent) was shown to induce recruitment of $\mathrm{NK}$ cells and $\mathrm{T}$ cells into the tumor 
while the numbers of MDSCs and regulatory $\mathrm{T}$ cells decreased [47]. When injected subcutaneously, these allogeneic DCs were shown to initiate host DC activation and migration to draining lymph node, leading to $\mathrm{T}$ cell activation. Injection of pro-inflammatory allogeneic DCs into a site (subcutaneous tissue or melanoma tumor) transfected with a virus vector, inducing local expression of the tumor antigen gp-100, resulted in the generation of gp-100 specific $\mathrm{CD}^{+} \mathrm{T}$ cells and when combined with transfer of gp100-specific pmel$1 \mathrm{~T}$ cells, a prolonged survival of B16-F10 melanoma-bearing mice was observed [47].

Based on these findings, we therefore expect that intratumorally injected pro-inflammatory allogeneic DCs (ilixadencel) in the clinical setting will induce recruitment of immune cells including NK-cells, DCs and T cells to the injection site. The cross-talk between the DCs and recruited NK cells will induce NK cell activation, subsequently leading to local tumor cell killing and release of cell-associated tumor antigens. NK-cell derived IFN- $\gamma$, in concert with TNF- $\alpha$ produced by the injected DCs and also by activated NK cells, will enhance cross-presentation of captured tumor antigens by recruited, endogenous, DCs. These antigen-loaded and cross-presenting DGs will start to mature due to activation by pro-inflammatory factors like TNF- $\alpha$ and IL- $1 \beta$ released by the injected allogeneic DCs. Production of IFN- $\gamma$ by recruited and subsequently activated NK cells and alloreactive $\mathrm{T}$ cells will furthermore favor the differentiation of Thl polarizing DCs [44]. Additionally, NK-cell and alloreactive T cell-derived IFN- $\gamma$ may inhibit immunosuppressive M2macrophages [48] and drive Treg fragility within the tumor [49]. The proposed mode of action for intratumorally injected ilixadencel is illustrated in Fig. 1.

\section{Toxicology Studies}

Intrarenal injection of allogeneic rat pro-inflammatory DCs (surrogate rat product) did not cause any severe changes in the clinical chemistry parameters investigated in the toxicology studies. Histological examination of major organs did not indicate any tissue damage or local accumulation of inflammatory cells (ectopic foci). Hematological parameters (mirroring bone marrow function) did not indicate bone marrow toxicity. These data indicated that no organ-specific autoimmune reactions developed and that potential seeding of cells from the administration site to remote organs did not induce local foci of inflammatory cell immune reactions that would reflect areas of in situ mixed leukocyte reaction induced by seeded viable DGs.

\section{Biodistribution Studies}

Radiolabelled $\left({ }^{111} \mathrm{In}\right)$ pro-inflammatory rat DCs (surrogate rat product) were injected intrarenally into a rat strain that is allogeneic to the DCs. No significant radioactivity signals were detected in the organs with a physiological barrier, such as the brain, gonads, skin and muscles. For the signals observed up to $72 \mathrm{~h}$ after administration in lungs, liver, spleen, bone marrow and kidneys (injected kidney as well as contralateral kidney), it was not evident if signals came from free or cell bound ${ }^{111} \mathrm{In}$. With the knowledge that injected DCs are not viable for more than 2 to 3 days after injection, and that histology of major organs did not indicate any tissue damage or local accumulation of inflammatory cells (ectopic foci), the signals detected in different organs, most likely distributed through passive transport by the bloodstream, might reflect ${ }^{111}$ In-labelled dead cells or cell debris.

\section{ILIXADENCEL DRUG PRODUCT}

The active substance ilixadencel (INN) is composed of monocyte-derived dendritic cells (MoDCs) that have been stimulated with a combination of activating factors (R848, Poly I:C and IFN- $\gamma$ ) ex vivo, resulting in a residual production of desirable pro-inflammatory factors (such as TNF- $\alpha$, IL-1 $\beta$ and MIP-1 $\beta$ and also high amounts of IL-12p70 and RANTES) and expression of co-stimulatory surface molecules (such as CD86) at the time of administration [46].

\section{Manufacturing}

The drug product is manufactured using a continuous process, starting with monocytes enriched from leukapheresis source material from a healthy donor and ending with a cryopreserved drug product, formulated in human plasma and DMSO $(10 \%(v / \mathrm{v}))$ at a concentration of $11.7 \times 10^{6}$ viable cells/mL. Drug product is filled into $2 \mathrm{~mL}$ polyolefin vials with chlorobutyl, Flurotec ${ }^{\circledR}$ coated, stoppers and aluminum crimp caps. It is stored in vapor phase nitrogen $\left(\leq-130^{\circ} \mathrm{C}\right)$ and is ready to use directly after thawing with no processing beyond extraction from the cells from the vial. The current shelf life of the product is 36 months when stored under real-time storage conditions (vapor phase nitrogen at $\leq-130^{\circ} \mathrm{C}$ ).

Using leukapheresis material collected from one donor, one batch of ilixadencel is sufficient to treat 25-50 patients with a two-dose regimen.

\section{Adventitious Agents Safety Evaluation}

The guidelines for CBMP (EMEA/CHMP/410869/2006, section 4.2.1) are followed, meaning that stringent sourcing requirements and acceptance criteria are in place for all raw materials and excipients derived from human sources. Donor screening and eligibility criteria are applied in accordance with country-specific law, EU directives (2006/17/EG and 2004/23/EG) and US requirements (CFR 1271.75). 


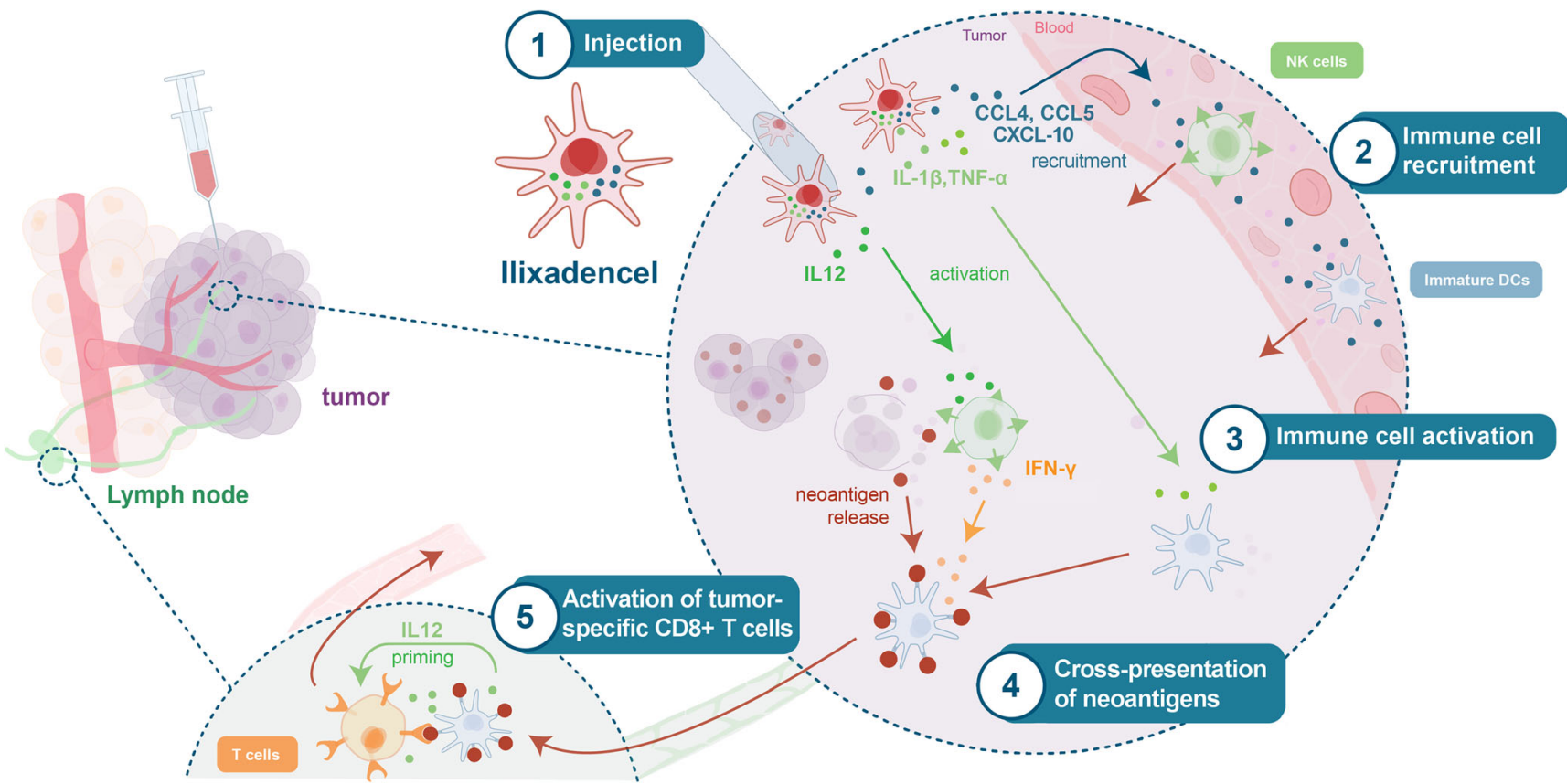

Fig. I Proposed mode of action for intratumorally injected ilixadencel. After administration into the viable part of the tumor, ilixadencel DCs will release ThI-associated chemokines, including CCL4, 5 and CXCLI 0 that recruit immune cells, including NK-cells and pre-DCs into the tumor. The interaction between recruited NK-cells and ilixadencel DCs induce NK-cell-mediated killing of adjacent tumor cells, resulting in release of tumor-associated antigens, including neoantigens. The production of IFN- $\gamma$ by activated NK-cells and TNF- $\alpha / \mathrm{L}-\mathrm{I} \beta$ released by ilixadencel DCs will induce maturation as well as increase crosspresentation of engulfed cell-associated tumor antigens by recruited endogenous "bystander" DCs. Migration of these antigen-loaded and matured "bystander"

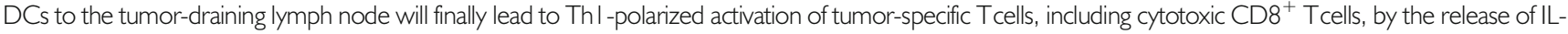
12.

Additional testing is applied in accordance with specific member state regulatory requirements. The risk of BSE/TSE transmission is considered negligible due to the use of permanent deferral criteria for donors, and the fact that no materials of bovine origin are used in the manufacturing process.

\section{REGULATORY ACTIVITIES}

In December 2015, WHO published, for the first time, a guideline on how to establish an International Nonproprietary Name (INN) for Gell Therapy Products (CTP) (INN Working Doc. 13.323 revision 4). The randomly chosen prefix (ilixa-) was to be followed by an infix describing cell type (-den-), for dendritic cells, and ended by the suffix (-cel) for all cell therapy products. To the best of our knowledge, Immunicum was one of the first companies that applied for an INN for a cell therapy product, and received in January 2017 the proposed recommended name ilixadencel (INN) from WHO that was later confirmed (WHO Drug Information, Vol. 31, No. 3, 2017 Recommended INN: List 78).

Regulation (EC) No 1394/2007 introduced a new type of product classification in Europe, Advanced Therapy Medicinal Products (ATMP). This regulation gave for the first time in Europe, clarity on how to classify cell- and gene therapy products and tissue engineered products. The European
Medicines Agency (EMA) established a special committee, the Committee for Advanced Therapies (CAT), to be involved in classification of, scientific advice for and review of these products. Immunicum applied for, and received in December 2015, classification by CAT/EMA for ilixadencel, as a somatic cell therapy medicinal product under Regulation $1394 / 2007$. In the US, cell therapy products are handled and reviewed by the Center for Biologics Evaluation and Research (CBER) division at the US Food and Drug Administration (FDA).

During development of any medicinal product, and in particular for cell therapy products being a relatively new group of therapies to treat patients, early interactions with regulatory authorities are very important. Both national authorities in Europe, EMA and FDA have established procedures for scientific advice and meetings during development. In the US, during the pre-submission stage and in dialogue with the FDA, Immunicum received guidance on the development plan, and the data and regulatory requirements for the Investigational New Drug (IND) submission. This was important for a successful IND submission and the IND clearance by FDA in December 2016 made it possible for the company to include US patients in the IM-201 (MERECA) study. Immunicum has also received EMA scientific advice and recently national advice from several regulatory authorities in EU on the development and design of study IM-202. 
The European Medicines Agency's Committee for Advanced Therapies (CAT) provides a certification procedure for ATMPs under development by micro, small and mediumsized enterprises (SMEs). This is an opportunity for SMEs to receive an assessment of the data generated at that stage in development, to check that they are on the right track for successful product development. The certification procedure involves the scientific evaluation of quality data and, when available, non-clinical data that SMEs have generated at any stage of the ATMP development process. Immunicum submitted data under this procedure to EMA and received in March 2018 the ATMP Certificate for Quality and Nonclinical Data for ilixadencel.

\section{CLINICAL STUDIES}

To date, two early clinical trials have been completed and two trials are currently active, as shown in Table I. The first-inhuman phase I open-label study was completed in 2014 and evaluated safety and immunologic response of ilixadencel in patients with newly diagnosed metastatic renal cell carcinoma (mRCG) (IM-101). The phase I open-label study, IM-102, tested ilixadencel alone and in combination with sorafenib in patients with advanced hepatocellular carcinoma (HCG). Recruitment is now complete in both the phase I study in gastrointestinal stromal tumors (GIST) (IM-103) and the multicenter comparative phase II study in patients with newly diagnosed mRCG (IM-201).

Overall, the safety profile observed across all trials is very good and is characterized by predominantly fever and chills as the treatment-related adverse events, and no DLT was found in doses up to $20 \times 10^{6}$ viable cells. Likewise, no evidence of autoimmunity or clinically significant allo-immunity was found in any trial. On the other hand, in both studies there was evidence of a tumor-specific immune response following treatment with ilixadencel. Finally, signs of antitumor activity were observed among patients with mRCG and advanced HCG in the completed trials.

\section{Phase I Study in Patients with Newly Diagnosed Metastatic RCC}

Twelve intermediate and poor risk patients with newly diagnosed synchronous metastatic renal cell carcinoma (mRCG) were included in the study (IM-101). A dose of 5-20 $\times 10^{6}$ of cells was injected into the renal tumor twice with 2 weeks interval before planned nephrectomy and the patients subsequently received standard of care [46]. No severe adverse events related to ilixadencel were observed. A massive infiltration of $\mathrm{CD}^{+} \mathrm{T}$ cells was found in 5 out of 12 removed kidney tumors (see Fig. 2). As illustrated in Fig. 3, the number and infiltration pattern of $\mathrm{CD}^{+} \mathrm{T}$ cells were nearly identical to the number and infiltration pattern of $\mathrm{CD} 3^{+} \mathrm{T}$ cells within tumor cell nests and by far outnumbered $\mathrm{CD} 4^{+} \mathrm{T}$ in tumor samples with high intratumoral T cells infiltration. In contrast, $\mathrm{CD} 4{ }^{+} \mathrm{T}$ cells generally predominated in the stroma surrounding tumor cell nests (Fig. 3b). The normal kidney tissue areas adjacent to tumor areas, including those adjacent to tumor areas with high $\mathrm{CD}^{+} \mathrm{T}$ cell infiltration, were generally only weakly infiltrated with $\mathrm{CD}^{+} \mathrm{T}$ cells (Fig. 3e). In the only evaluable metastatic lesion surgically removed (subcutaneous lesion removed 1 month after nephrectomy) from a patient with massive $\mathrm{CD}^{+} \mathrm{T}$ cell infiltration in the primary kidney tumor, a similar massive infiltration of $\mathrm{CD}^{+} \mathrm{T}$ cells was observed (Fig. 3f). Moreover, high $\mathrm{CD}^{+}$infiltration was generally associated with strong HLA-DR expression in adjacent tumor cells (Fig. 3d).

No objective tumor response was observed in any patient at 3 months follow up after nephrectomy and 6 out of 11 evaluable patients subsequently received additional treatment with standard tyrosine kinase inhibitors (TKI) at progression. Three of these 6 patients experienced an objective tumor response, including one sunitinib-treated patient, showing strong intratumoral infiltration of $\mathrm{CD}^{+} \mathrm{T}$ cells in the removed tumor, who responded with a complete and durable regression ( $>52$ months, still ongoing) of 4 brain metastases (and also all liver metastases) (Fig. 4). This highly favourable long-lasting response was unexpected since mRCC-derived CNS metastases are not expected to respond to sunitinib treatments [50]; it was hypothesized that the add-on treatment with sunitinib inhibited the tumor-associated immunosuppression by inhibiting MDSCs [7, 51] thereby opening up for a $\mathrm{T}$ cell-mediated tumor destruction. Median overall survival (mOS) for the whole patient group was 48.0 months and three patients have passed 5 years survival. These survival data are encouraging in the light of historical data in patients with recently diagnosed (including synchronous) $\mathrm{mRCC}$ patients treated with VEGF-targeted therapies, including sunitinib, where a median OS of 15-16 months has been reported $[52,53]$.

\section{Phase I Study in Patients with Advanced HCC}

This was a single arm, open-label, phase I trial including 18 patients with advanced liver cancer (IM-102), 17 patients with unresectable hepatocellular carcinoma $(\mathrm{HCG})$ and 1 patient with advanced cholangiocarcinoma $(\mathrm{CG})$. Patients were to be treated with three separate injections of ilixadencel (10 or $20 \times 10^{6}$ of cells) directly into their tumor (at approximately day 1, 14 and 42) and patients were followed for 6 months after last injection. The final patient disposition was as follows: 7 patients were treated with ilixadencel as second line systemic treatment, 10 patients were treated as first line treatment of which 6 patients were treated in combination with sorafenib in the first line setting. 
Table I Overview of the Clinical Trials with llixadencel

\begin{tabular}{lllllll}
\hline Study No & ClinicalTrials.gov identifier & Phase & Indication & Site(s) & Patients planned & Status \\
\hline IM-I0I & NCT0I5250I7 & I & mRCC & Uppsala University Hospital, Sweden & 12 & Completed \\
IM-I02 & NCT0I97466I & I & HCC & Sahlgrenska University Hospital, Sweden & 18 & Completed \\
IM-20I & NCT02432846 & II & mRCC & Multicenter in Europe and US & 88 & Active, and recruitment completed \\
IM-I03 & NCT02686944 & I & GIST & Karolinska University Hospital, Sweden & Appr. I2 & Active, and recruitment completed \\
\hline
\end{tabular}

There were no life-threatening or fatal treatment-related adverse events (AEs). Overall, with only one exception, all treatment-related AEs were mild-to-moderate. The most common treatment-related AEs were described as fever and/or chills and could be easily managed. The exception was one patient receiving ilixadencel and sorafenib as combination therapy, who presented with a suspected sepsis event (grade 3) that subsequently recovered. As a reference, current standard of care, such as sorafenib or regorafenib, report in the literature [54] and in prescribing information severe (grade 3) drug-related AEs in at least one in three HCC patients treated.

Evidence of systemic immunological response to the treatment, as measured by an increase in $\alpha$-fetoprotein and/or hTERT specific, IFN- $\gamma$ producing $\mathrm{CD}^{+} \mathrm{T}$ cells in the blood, was demonstrated in 9 out of 13 evaluable patients $(69 \%)$. Overall survival ranged between 1.6-21.4 months in the total group of $17 \mathrm{HCC}$ patients at closure of study with three patients still alive. More in-depth group and patient analyses are ongoing.

The patient with advanced cholangiocarcinoma was included by a study amendment and received three intratumoral administrations of ilixadencel, each dose administered the day after transarterial chemo-embolization (TACE) with doxorubicin-eluting microspheres, aimed at inducing immunogenic tumor-cell death within the ilixadencel-treated tumor lesion. The frequency of IFN- $\gamma$ producing peripheral blood $\mathrm{CD}^{+} \mathrm{T}$ cells against hTERT and AFP was increased 1 week after the third dose of ilixadencel when compared to baseline levels. The patient's tumor showed a partial response at 3 months. At 6 months the tumor progressed and standard gemcitabine/cisplatine $(\mathrm{G} / \mathrm{G})$ regimen was started, which resulted in a nearly complete tumor response. Overall, this patient survived for 41 months post enrolment. These survival data are encouraging when comparing to historical data for
Fig. 2 Micrographs illustrating immunohistochemical staining of tissue samples from all 12 surgically removed primary renal RCC tumors with anti-CD8 antibodies. The surgical removal of the tumorbearing kidney was performed I2 weeks after the second (last) administration of ilixadencel. Original magnification $\times 100$. The data in figure originates from Laurell et al. [46].
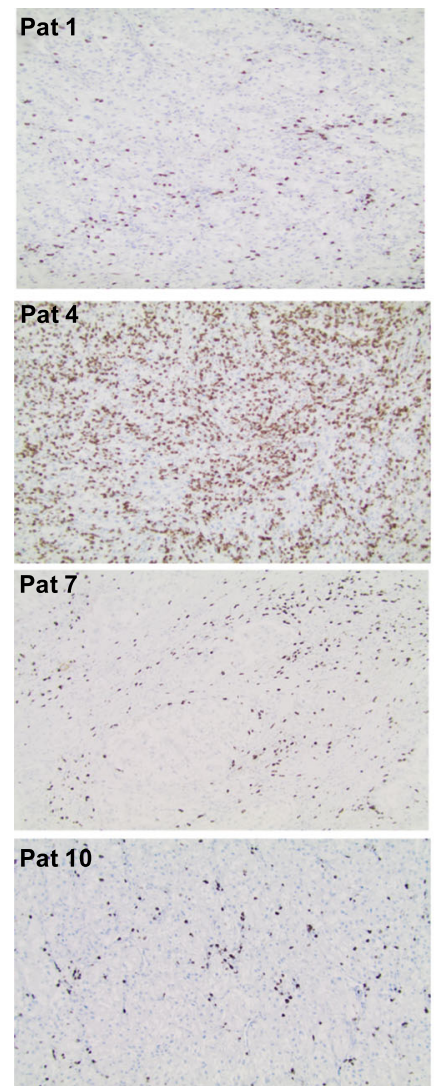
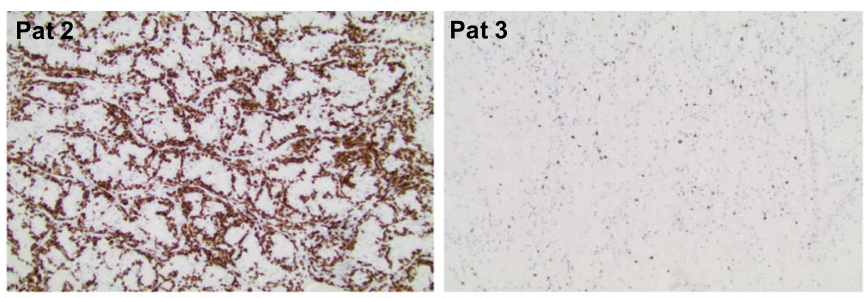

\section{Pat 5.}
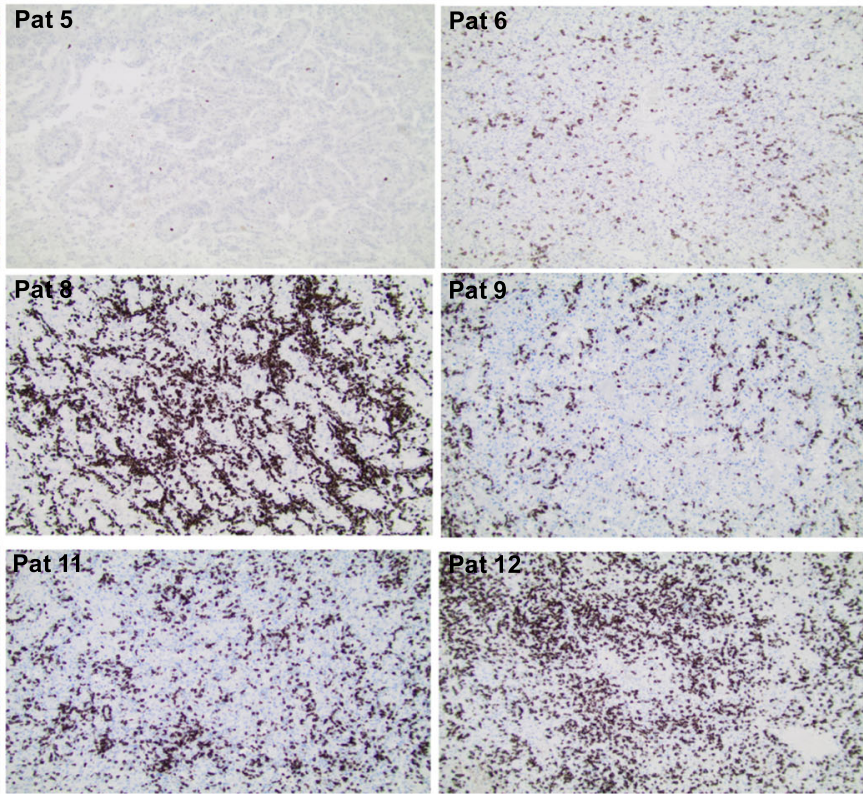
Fig. 3 Micrographs illustrating immunohistochemical staining of tissue samples from surgically removed primary renal RCC tumors (surgically removed I2 weeks after the second (last) administration of ilixadencel) in consecutive sections from one RCC tumor with high $\mathrm{CD}^{+}$Tcell infiltration (A-D, all from patient 2). (E) illustrates $\mathrm{CD}^{+}{ }^{+}$T cells in normal kidney parenchyma adjacent to tumor areas from patient 2 and (F) illustrates $\mathrm{CD}^{+}{ }^{+}$Tcell infiltration in a subcutaneous metastatic lesion from patient 2. Original magnification $\times 100$. The data in figure originates from Laurell et al. [46].

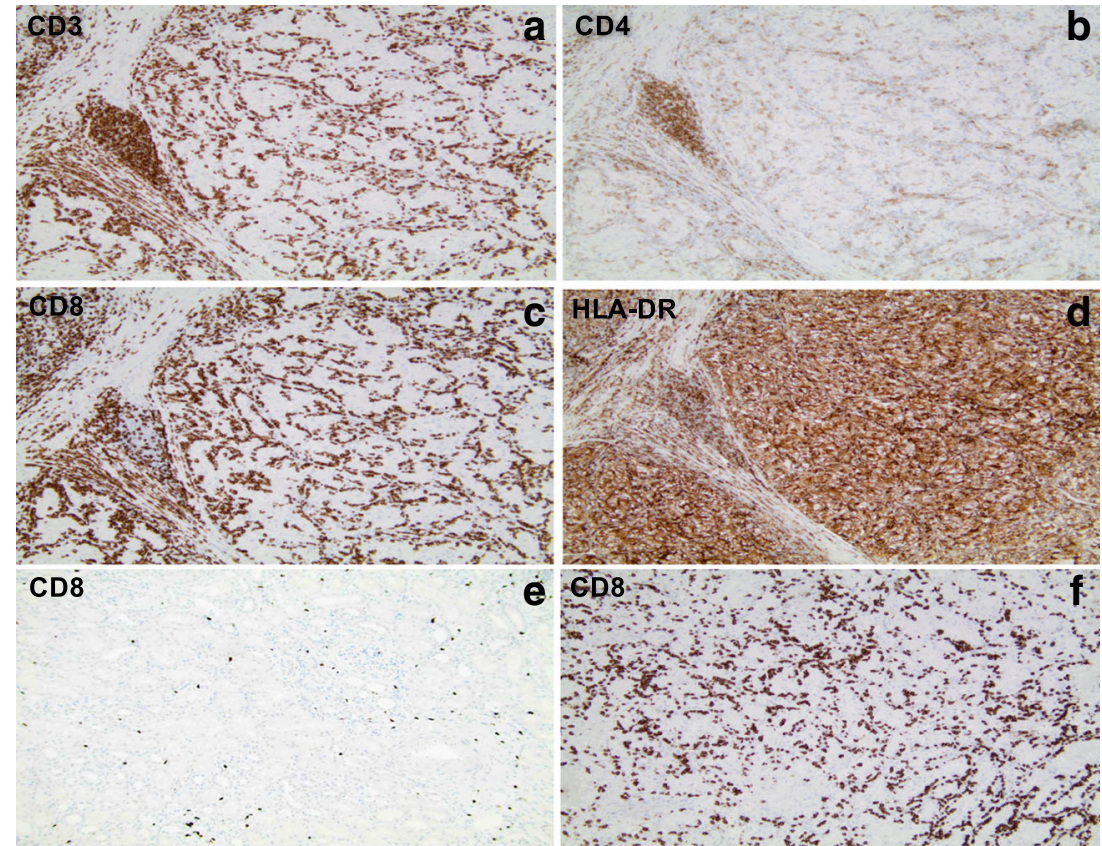

patients with advanced $\mathrm{CC}$ on standard $\mathrm{G} / \mathrm{C}$ treatment with an expected overall survival of 12 months [55]. We therefore hypothesize that combination of ilixadencel-induced recruitment and activation of endogenous DGs, local doxorubicininduced enhancement of immunogenic cell death as well as the addition of gemcitabine that is known to deplete MDSCs $[7,56]$ may act in synergy as indicated by objective tumor response and long survival.

\section{Phase I Study in Patients with Gastrointestinal Stromal Tumors}

This study is a prospective single arm, open-label phase I safety study of ilixadencel in patients with progressing gastrointestinal stromal tumors (GIST) (IM-103) during ongoing second, third or fourth line treatment with tyrosine kinase inhibition therapy. A total of 6 patients have been enrolled;
Fig. 4 CT-scans from a patient with CNS and liver metastases 4 months after start of ilixadencel treatment but before additional treatment with sunitinib (A and $C$, respectively) and 6 months (B) or 12 months (D) after start of sunitinib treatment. The data in figure originates from Laurell et al. [46].
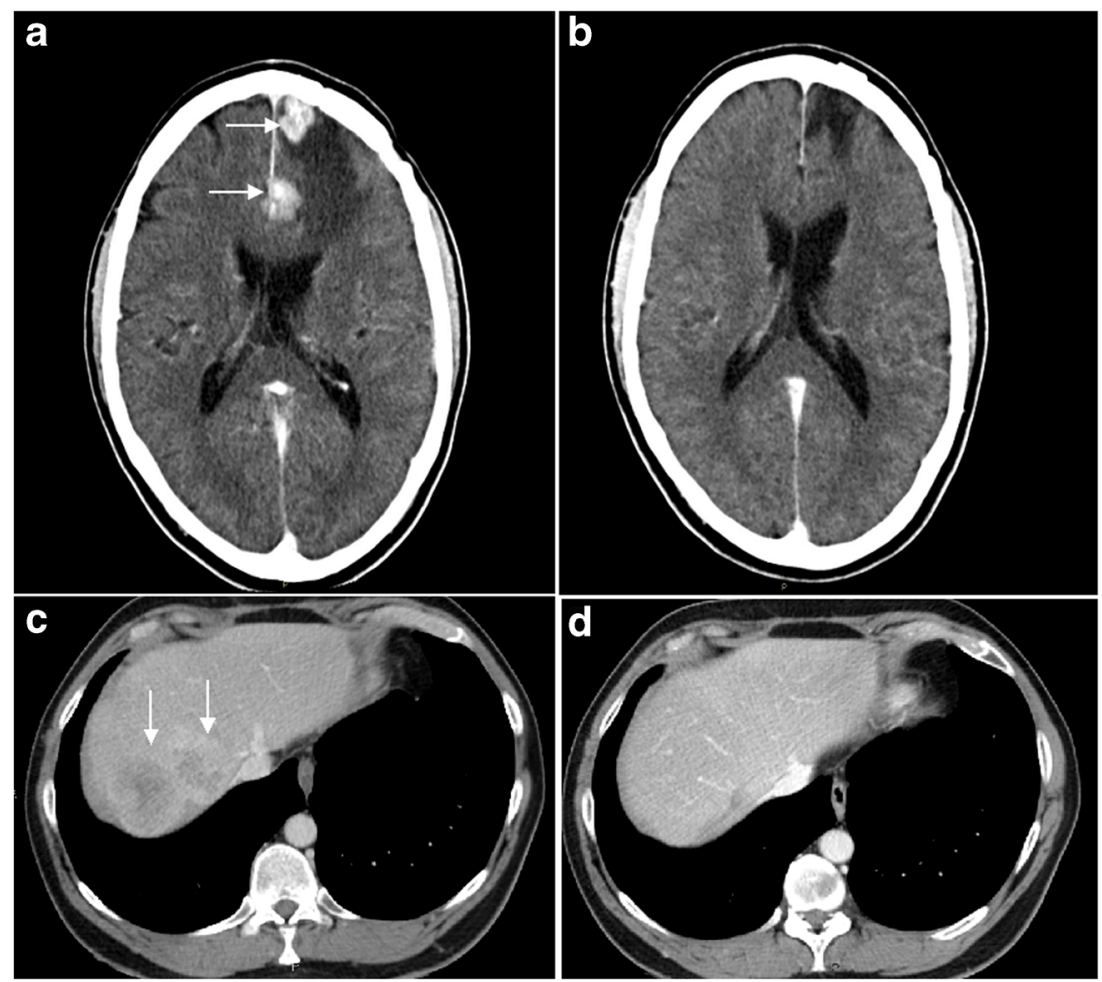
the analysis of primary outcome measures of safety and tolerability, as well as initial secondary outcomes on efficacy, tumor response and progression-free survival, are expected in the course of 2019.

\section{Phase II Study IM-20 I (MERECA) with Ilixadencel Pre-Nephrectomy Followed by Sunitinib Post-Nephrectomy Compared with Standard Treatment with Sunitinib Monotherapy Post-Nephrectomy}

Based on data from the initial phase I trial in patients with newly diagnosed $\mathrm{mRCC}$, suggesting that ilixadencel treatment followed by standard sunitinib treatment may act synergistically, a phase II mRCC study was initiated. This study is an open-label, randomized study comparing ilixadencel prenephrectomy, followed by sunitinib treatment with only sunitinib post-nephrectomy. Patient recruitment has been completed and a total of 88 newly diagnosed mRCA patients have been randomized into the trial conducted at 28 centers in eight European countries and the US. The 18-month follow-up period for the last patient treated will be completed around mid-year 2019.

\section{Planned Phase Ib/II Study Multi-Indication Trial IM-202 Including Head and Neck, Lung and Gastric Cancer in Combination with a PD-I/PD-LI Inhibitor}

Based on the potential synergy observed between ilixadencel and drugs that inhibit immunosuppression (sunitinib and gemcitabine), including unpublished preclinical data indicating that ilixadencel and anti-PD- 1 treatment have a synergistic anti-tumor effect in the CT-26 mouse tumor model, a new combination study is planned: this is a randomized, open-label, multicenter, phase Ib/II trial evaluating the safety and efficacy of intratumorally-administered ilixadencel in combination with checkpoint inhibitor (CPI) in advanced cancer subjects who are candidates for CPI therapy. The indications will be advanced (unresectable or metastatic) head and neck squamous-cell carcinoma of the (HNSCG), non-small cell lung cancer (NSCLC), and gastric or gastroesophageal junction (GEJ) adenocarcinoma.

\section{SUMMARY}

The cross-presenting function of DCs is essential for the therapeutic effect of CPIs, but the baseline CTL-priming function is suboptimal. These observations suggest the potential to devise intratumoral (in situ) administration of immune primers to enhance cross-presentation of cell-associated tumor antigens, including neoantigens, and thereby increase the efficacy of CPIs. Based on our preclinical and early clinical findings, the intratumoral administration of ilixadencel in the clinical setting is expected to induce a cascade of immunological events, including recruitment of NK cells, DCs and T cells that ultimately leading to desirable Th1-polarized activation of DCs and efficient MHC class I cross-presentation of cellassociated tumor antigens. Clinical data are further suggesting that the combination of ilixadencel and drugs reducing tumor-derived immunosuppression may have a synergistic antitumor effect.

Acknowledgments and Disclosures. AKP, JK, EH, SZ, SL and PS report ownership of stocks in Immunicum AB and are all Immunicum employees. MJ report ownership of stocks in Immunicum $\mathrm{AB}$ and is a consultant to Immunicum $\mathrm{AB}$.

Data Availability. Data sharing not applicable to this article as no datasets were generated or analysed during the current study.

Open Access This article is distributed under the terms of the Creative Commons Attribution 4.0 International License (http://creativecommons.org/licenses/by/4.0/), which permits unrestricted use, distribution, and reproduction in any medium, provided you give appropriate credit to the original author(s) and the source, provide a link to the Creative Commons license, and indicate if changes were made.

\section{REFERENCES}

1. Melief CJ, van Hall T, Arens R, Ossendorp F, van der Burg SH. Therapeutic cancer vaccines. J Clin Invest. 2015;125(9):3401-12.

2. Ock CY, Keam B, Kim S, Lee JS, Kim M, Kim TM, et al. PanCancer Immunogenomic perspective on the tumor microenvironment based on PD-L1 and CD8 T-cell infiltration. Clinical cancer research : an official journal of the American Association for Cancer Research. 2016;22(9):2261-70.

3. Gubin MM, Zhang X, Schuster H, Caron E, Ward JP, Noguchi T, et al. Checkpoint blockade cancer immunotherapy targets tumourspecific mutant antigens. Nature. 2014;515(7528):577-81.

4. Karaki S, Anson M, Tran T, Giusti D, Blanc C, Oudard S, et al. Is there still room for Cancer vaccines at the era of checkpoint inhibitors. Vaccines (Basel) 2016;4(4):37. https://doi.org/10.3390/ vaccines 4040037 .

5. Spranger S, Luke JJ, Bao R, Zha Y, Hernandez KM, Li Y, et al. Density of immunogenic antigens does not explain the presence or absence of the T-cell-inflamed tumor microenvironment in melanoma. Proc Natl Acad Sci U S A. 2016;1 13(48):E7759-E68.

6. Gajewski TF. The next hurdle in Cancer immunotherapy: overcoming the non-T-cell-inflamed tumor microenvironment. Semin Oncol. 2015;42(4):663-71.

7. Draghiciu O, Nijman HW, Hoogeboom BN, Meijerhof T, Daemen T. Sunitinib depletes myeloid-derived suppressor cells and synergizes with a cancer vaccine to enhance antigen-specific immune responses and tumor eradication. Oncoimmunology. 2015;4(3):e989764. 
8. Robbins PF, Lu YC, El-Gamil M, Li YF, Gross C, Gartner J, et al. Mining exomic sequencing data to identify mutated antigens recognized by adoptively transferred tumor-reactive T cells. Nat Med. 2013;19(6):747-52.

9. van Rooij N, van Buuren MM, Philips D, Velds A, Toebes M, Heemskerk B, et al. Tumor exome analysis reveals neoantigenspecific T-cell reactivity in an ipilimumab-responsive melanoma. Journal of clinical oncology : official journal of the American Society of Clinical Oncology. 2013;31(32):e439-42.

10. Schumacher TN, Schreiber RD. Neoantigens in cancer immunotherapy. Science. 2015;348(6230):69-74

11. Fritsch EF, Hacohen N. Wu CJ. Personal neoantigen cancer vaccines: the momentum builds. Oncoimmunology. 2014;3:e29311.

12. Gilboa E. The makings of a tumor rejection antigen. Immunity. 1999;11(3):263-70.

13. Sahin U, Derhovanessian E, Miller M, Kloke BP, Simon P, Lower $\mathrm{M}$, et al. Personalized RNA mutanome vaccines mobilize polyspecific therapeutic immunity against cancer. Nature. 2017;547(7662):222-6.

14. Ott PA, Hu Z, Keskin DB, Shukla SA, Sun J, Bozym DJ, et al. An immunogenic personal neoantigen vaccine for patients with melanoma. Nature. 2017;547(7662):217-21.

15. Hammerich L, Binder A, Brody JD. In situ vaccination: Cancer immunotherapy both personalized and off-the-shelf. Mol Oncol. 2015;9(10):1966-81.

16. Palucka AK, Coussens LM. The basis of Oncoimmunology. Cell. 2016;164(6):1233-47.

17. Noy R, Pollard JW. Tumor-associated macrophages: from mechanisms to therapy. Immunity. 2014;41(1):49-61.

18. Tauriello DVF, Palomo-Ponce S, Stork D, Berenguer-Llergo A, Badia-Ramentol J, Iglesias M, et al. TGFbeta drives immune evasion in genetically reconstituted colon cancer metastasis. Nature. 2018;554(7693):538-43.

19. Mariathasan S, Turley SJ, Nickles D, Castiglioni A, Yuen K, Wang $\mathrm{Y}$, et al. TGFbeta attenuates tumour response to PD-L1 blockade by contributing to exclusion of T cells. Nature. 2018;554(7693): 544-8.

20. Spranger S, Dai D, Horton B, Gajewski TF. Tumor-residing Batf3 dendritic cells are required for effector $\mathrm{T}$ cell trafficking and adoptive T cell therapy. Cancer Cell. 2017;31(5):711-23. e4

21. Broz ML, Binnewies M, Boldajipour B, Nelson AE, PollackJL, Erle DJ, et al. Dissecting the tumor myeloid compartment reveals rare activating antigen-presenting cells critical for $\mathrm{T}$ cell immunity. Cancer Cell. 2014;26(5):638-52.

22. Salmon H, Idoyaga J, Rahman A, Leboeuf M, Remark R, Jordan S, et al. Expansion and activation of CD103(+) dendritic cell progenitors at the tumor site enhances tumor responses to therapeutic PD-L1 and BRAF inhibition. Immunity. 2016;44(4):924-38.

23. Roberts EW, Broz ML, Binnewies M, Headley MB, Nelson AE, Wolf DM, et al. Critical role for CD103(+)/CD141(+) dendritic cells bearing CCR7 for tumor antigen trafficking and priming of $\mathrm{T}$ cell immunity in melanoma. Cancer Cell. 2016;30(2):324-36.

24. Ruffell B, Chang-Strachan D, Chan V, Rosenbusch A, Ho CM, Pryer N, et al. Macrophage IL-10 blocks CD8+ T cell-dependent responses to chemotherapy by suppressing IL-12 expression in intratumoral dendritic cells. Cancer Cell. 2014;26(5):623-37.

25. Bottcher JP, Bonavita E, Chakravarty P, Blees H, CabezaCabrerizo M, Sammicheli S, et al. NK cells stimulate recruitment of cDCl into the tumor microenvironment promoting Cancer immune control. Cell. 2018;172(5):1022-37. e14

26. Vieira PL, de Jong EC, Wierenga EA, Kapsenberg ML, Kalinski P. Development of Thl-inducing capacity in myeloid dendritic cells requires environmental instruction. Journal of immunology (Baltimore, Md : 1950). 2000;164(9):4507-12.

27. Fonteneau JF, Kavanagh DG, Lirvall M, Sanders C, Cover TL, Bhardwaj N, et al. Characterization of the MHC class I cross- presentation pathway for cell-associated antigens by human dendritic cells. Blood. 2003;102(13):4448-55.

28. Joffre OP, Segura E, Savina A, Amigorena S. Cross-presentation by dendritic cells. Nat Rev Immunol. 2012;12(8):557-69.

29. Deauvieau F, Ollion V, Doffin AC, Achard C, Fonteneau JF, Verronese E, et al. Human natural killer cells promote crosspresentation of tumor cell-derived antigens by dendritic cells. Int J Cancer. 2015;136(5):1085-94.

30. Srivastava RM, Trivedi S, Concha-Benavente F, Gibson SP, Reeder C, Ferrone S, et al. CD137 stimulation enhances Cetuximab-induced natural killer: dendritic cell priming of antitumor T-cell immunity in patients with head and neck Cancer. Clinical cancer research : an official journal of the American Association for Cancer Research. 2017;23(3):707-16.

31. Srivastava RM, Lee SC, Andrade Filho PA, Lord CA, Jie HB, Davidson HC, et al. Cetuximab-activated natural killer and dendritic cells collaborate to trigger tumor antigen-specific T-cell immunity in head and neck cancer patients. Glinical cancer research : an official journal of the American Association for Cancer Research. 2013;19(7):1858-72.

32. Lopez-Albaitero A, Mailliard R, Hackman T, Andrade Filho PA, Wang X, Gooding W, et al. Maturation pathways of dendritic cells determine TAP1 and TAP2 levels and cross-presenting function. J Immunother. 2009;32(5):465-73.

33. Kratky W, Reis e Sousa C, Oxenius A, Sporri R. Direct activation of antigen-presenting cells is required for CD8+ T-cell priming and tumor vaccination. Proc Natl Acad Sci U S A. 2011;108(42): 17414-9.

34. Sanchez-Paulete AR, Teijeira A, Cueto FJ, Garasa S, Perez-Gracia JL, Sanchez-Arraez A, et al. Antigen cross-presentation and T-cell cross-priming in cancer immunology and immunotherapy. Ann Oncol. 2017;28(suppl_12):xii74.

35. Liu C, Lou Y, Lizee G, Qin H, Liu S, Rabinovich B, et al. Plasmacytoid dendritic cells induce NK cell-dependent, tumor antigen-specific $\mathrm{T}$ cell cross-priming and tumor regression in mice. J Clin Invest. 2008;118(3):1165-75.

36. Yewdall AW, Drutman SB, Jinwala F, Bahjat KS, Bhardwaj N. CD8+ $\mathrm{T}$ cell priming by dendritic cell vaccines requires antigen transfer to endogenous antigen presenting cells. PLoS One. 2010;5(6):e11144.

37. Bosnjak L, Miranda-Saksena M, Koelle DM, Boadle RA, Jones CA, Cunningham AL. Herpes simplex virus infection of human dendritic cells induces apoptosis and allows cross-presentation via uninfected dendritic cells. Journal of immunology (Baltimore, Md : 1950). 2005;174(4):2220-7.

38. Smed-Sorensen A, Chalouni C, Chatterjee B, Cohn L, Blattmann $\mathrm{P}, \mathrm{Nakamura} \mathrm{N}$, et al. Influenza a virus infection of human primary dendritic cells impairs their ability to cross-present antigen to CD8 T cells. PLoS Pathog. 2012;8(3):e1002572.

39. Pang IKI, Iwasaki T. A. IL-1R signaling in dendritic cells replaces pattern-recognition receptors in promoting CD8(+) T cell responses to influenza a virus. Nat Immunol. 2013;14(3):246-53.

40. Silvin A, Yu CI, Lahaye X, Imperatore F, Brault JB, Cardinaud S, et al. Constitutive resistance to viral infection in human CD141(+) dendritic cells. Sci Immunol. 2017;2(13):eaai8071. https://doi.org/ 10.1126/sciimmunol.aai8071.

41. Napolitani G, Rinaldi A, Bertoni F, Sallusto F, Lanzavecchia A. Selected toll-like receptor agonist combinations synergistically trigger a $\mathrm{T}$ helper type 1-polarizing program in dendritic cells. Nat Immunol. 2005;6(8):769-76.

42. Gustafsson K, Ingelsten M, Bergqvist L, Nystrom J, Andersson B, Karlsson-Parra A. Recruitment and activation of natural killer cells in vitro by a human dendritic cell vaccine. Cancer Res. 2008;68(14):5965-71. 
43. Muthuswamy R, Urban J, LeeJJ, Reinhart TA, Bartlett D, Kalinski P. Ability of mature dendritic cells to interact with regulatory $\mathrm{T}$ cells is imprinted during maturation. Cancer Res. 2008;68(14):5972-8.

44. Wallgren AC, Andersson B, Backer A, Karlsson-Parra A. Direct allorecognition promotes activation of bystander dendritic cells and licenses them for Thl priming: a functional link between direct and indirect allosensitization. Scand J Immunol. 2005;62(3):234-42.

45. Fotaki G, Jin C, Ramachandran M, Kerzeli IK, Karlsson-Parra A, Yu D, et al. Pro-inflammatory allogeneic DCs promote activation of bystander immune cells and thereby license antigen-specific Tcell responses. Oncoimmunology. 2018;7(3):e1395126.

46. Laurell A, Lonnemark M, Brekkan E, Magnusson A, Tolf A, Wallgren AC, et al. Intratumorally injected pro-inflammatory allogeneic dendritic cells as immune enhancers: a first-in-human study in unfavourable risk patients with metastatic renal cell carcinoma. Journal for immunotherapy of cancer. 2017;5:52.

47. Fotaki G, Jin C, Kerzeli IK, Ramachandran M, Martikainen MM, Karlsson-Parra A, et al. Cancer vaccine based on a combination of an infection-enhanced adenoviral vector and pro-inflammatory allogeneic DCs leads to sustained antigen-specific immune responses in three melanoma models. Oncoimmunology. 2018;7(3): e1397250.

48. Duluc D, Corvaisier M, Blanchard S, Catala L, Descamps P, Gamelin E, et al. Interferon-gamma reverses the immunosuppressive and protumoral properties and prevents the generation of human tumor-associated macrophages. Int J Cancer. 2009;125(2): 367-73.

49. Overacre-Delgoffe AE, Chikina M, Dadey RE, Yano H, Brunazzi EA, Shayan G, et al. Interferon-gamma drives Treg fragility to promote anti-tumor immunity. Cell. 2017;169(6):1130-41. el1

50. Escudier B, Porta C, Schmidinger M, Algaba F, Patard JJ, Khoo V, et al. Renal cell carcinoma: ESMO clinical practice guidelines for diagnosis, treatment and follow-up. Ann Oncol. 2014;25(Suppl 3): iii49-56.

51. Ko JS, Zea AH, Rini BI, Ireland JL, Elson P, Cohen P, et al. Sunitinib mediates reversal of myeloid-derived suppressor cell accumulation in renal cell carcinoma patients. Clinical cancer research : an official journal of the American Association for Cancer Research. 2009;15(6):2148-57.

52. Heng DY, Xie W, Regan MM, Warren MA, Golshayan AR, Sahi C, et al. Prognostic factors for overall survival in patients with metastatic renal cell carcinoma treated with vascular endothelial growth factor-targeted agents: results from a large, multicenter study. Journal of clinical oncology : official journal of the American Society of Clinical Oncology. 2009;27(34):5794-9.

53. Ko JJ, Choueiri TK, Rini BI, Lee JL, Kroeger N, Srinivas S, et al. First-, second-, third-line therapy for mRCC: benchmarks for trial design from the IMDC. Br J Cancer. 2014;110(8):1917-22.

54. Bruix J, Qin S, Merle P, Granito A, Huang YH, Bodoky G, et al. Regorafenib for patients with hepatocellular carcinoma who progressed on sorafenib treatment (RESORCE): a randomised, double-blind, placebo-controlled, phase 3 trial. Lancet. 2017;389(10064):56-66.

55. Valle J, Wasan H, Palmer DH, Cunningham D, Anthoney A, Maraveyas A, et al. Cisplatin plus gemcitabine versus gemcitabine for biliary tract cancer. N Engl J Med. 2010;362(14):1273-81.

56. Suzuki E, Kapoor V, Jassar AS, Kaiser LR, Albelda SM. Gemcitabine selectively eliminates splenic gr-1+/CD1 lb+ myeloid suppressor cells in tumor-bearing animals and enhances antitumor immune activity. Clinical cancer research : an official journal of the American Association for Cancer Research. 2005; 1 1(18): 6713-21. 